\title{
Integral stellar-nebular model of NGC 7009
}

\author{
Celia R. Fierro ${ }^{1}$, Antonio Peimbert ${ }^{1}$, Leonid Georgiev ${ }^{1}$, \\ Christophe Morisset ${ }^{1,2}$ and Anabel Arrieta ${ }^{3}$ \\ ${ }^{1}$ Instituto de Astronomía, Universidad Nacional Autónoma de México, \\ Apdo. Postal 70-264, México D.F, C.P. 04510, México \\ email: crfierro@astro.unam.mx, antonio@astroscu.unam.mx, georgiev@astro.unam.mx, \\ chris.morisset@gmail.com, and anabel.arrieta@uia.mx \\ ${ }^{2}$ Instituto de Astrofísica de Canarias, E-38200, La Laguna, Tenerife, Spain.
}

${ }^{3}$ Universidad Iberoamericana, Departamento de Física y Matemáticas, Avenida Prolongacion Paseo de la Reforma 880, Lomas de Santa Fe, CP 01210, México, DF, México

\begin{abstract}
We developed a self-consistent stellar-nebular model for NGC 7009. This model reproduces the available observations ranging from optical to UV. The combined approach to the modeling process produces more constraints and thus more trustworty results. After obtaining the model, we perform a comparative study of the chemical composition of $\mathrm{He}, \mathrm{N}, \mathrm{O}, \mathrm{Ne}, \mathrm{Cl}$, and $\mathrm{S}$ in the nebula and its central star. Concluding that the stellar composition agrees with the nebular composition with $t^{2} \neq 0.00$.
\end{abstract}

Keywords. stars: abundances, stars: atmospheres, stars: fundamental parameters, stars: rotation, stars: winds, outflows, ISM: abundances, planetary nebulae: individual (NGC 7009)

\section{Stellar-nebular model}

A preliminary model of the star was obtaining fitting the lines and P-Cygni profiles in the UV and the visual spectra. A preliminary model of the PN was obtained fitting the intensities of the nebular lines. The stellar model was used as ionization source for the nebular one. The stellar models were calculed with the CMFGEN code (Hiller \& Miller 1998). The nebular models were calculed with CLOUDY (Ferland et al. 1998). The parameters in the models were scaled to the same distance. We perform several iterations in order to obtain a stellar-nebular model which reproduces simultaneosly the observations of the nebula and the star

\section{Determination of the distance using evolutionary tracks}

In order to constraint the distance, we use the evolutionary tracks of Vassiliadis \& Wood (1994). Each point in the evolutionary tracks is a combination of $T_{\text {eff }}, L$, and time of evolution. Any stellar-nebular model valid for the object should be within the area bounded by the $T_{\text {eff }}$ of the star and the kinematic age range of the nebula. We measured $v_{\text {exp }}=20 \pm 2 \mathrm{~km} / \mathrm{s}$ in the central region of the nebula from the [O III] $\lambda 5007$ line. With our $T_{\text {eff }}=86000 \pm 5000 \mathrm{~K}$ (Table 1 ) and $2150 \leqslant \tau_{\text {kin }} \leqslant 8550 \mathrm{yr}$ we delimited

Table 1. Parameters of the integral stellar-nebular model.

\begin{tabular}{llll}
\hline$T_{\text {eff }}$ & $86000 \pm 5000 \mathrm{~K}$ & $v_{\infty}$ & $2650 \mathrm{~km} \mathrm{~s}^{-1}$ \\
$L$ & $5500 \pm 500 \mathrm{~L} \odot$ & $v \sin i$ & $110 \pm 20 \mathrm{~km} \mathrm{~s}^{-1}$ \\
$\log g$ & $5.3 \pm 2$ & $\tau_{\text {kin }}$ & $5000 \pm 1500 \mathrm{yrs}$ \\
Distance & $1.4 \pm 0.4 \mathrm{kpc}$ & Size & $15 \operatorname{arc~sec}$ \\
$R_{\text {star }}$ & $0.33 \pm 0.2 \mathrm{R} \odot$ & $R_{\text {in }}$ & $4.34 \times 10^{16} \mathrm{~cm}$ \\
$M_{\text {star }}$ & $0.7 \pm 0.1 \mathrm{M}_{\odot}$ & $R_{\text {out }}$ & $2.60 \times 10^{17} \mathrm{~cm}$ \\
$M_{\text {progenitor }}$ & $1.6 \pm 0.2 \mathrm{M}_{\odot}$ & Filling factor & 0.7 \\
$\dot{M}$ & $8 \times 10^{-10} \mathrm{M}_{\odot} \mathrm{yr}^{-1}$ & & \\
\hline
\end{tabular}




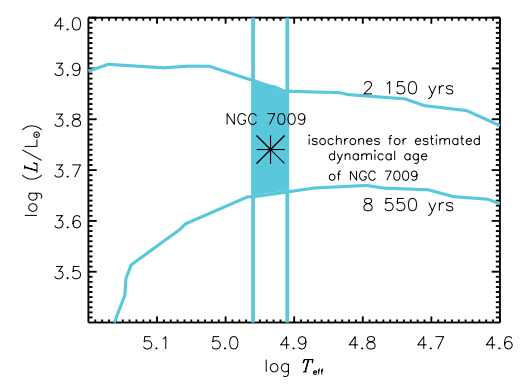

Figure 1. Region of solution is marked.

the permitted region in the $\mathrm{H}-\mathrm{R}$ diagram. Figure 1 shows the limits of the luminosity and the position of our best stellar-nebular model.

\section{Stellar and nebular chemical composition}

For the comparative study of the nebular and stellar chemical composition, we define two regions in the nebula: High and Medium, by reference to the ionization degree (ratio $\left[\begin{array}{ll}\mathrm{O} & \mathrm{III}\end{array}\right] /[\mathrm{O} \mathrm{II}]$ ). We calculed the abundances of $\mathrm{He}, \mathrm{N}, \mathrm{O}, \mathrm{Ne}, \mathrm{Cl}$ and $\mathrm{S}$ for the nebula under assumption of homogeneous temperature $\left(t^{2}=0.00\right)$ and under assumption of temperature fluctuations, $t^{2}=0.084$ and 0.113 for the High and Medium regions, respectively (hereafter $t^{2} \neq 0.00$ ). The nebular model was tuned to reproduce the observed recombination lines of $\mathrm{He}, \mathrm{N}, \mathrm{O}$ and the collisional excited lines of $\mathrm{N}, \mathrm{O}, \mathrm{Ne}, \mathrm{Cl}$ and $\mathrm{S}$.

The abundances of He and $\mathrm{N}$ obtained from RLs in the nebular model agree with the stellar model and CELS assuming temperature fluctuations $\left(\mathrm{t}^{2} \neq 0.00\right)$.

The nebular abundances are consistent with homogeneous chemical composition in the studied regions. The He, $\mathrm{O}$ and Ne abundances are higher than solar. The $\mathrm{N}, \mathrm{Cl}$ and $\mathrm{S}$ abundances are close to the solar value (see Figure 2). The abundances of He, $\mathrm{N}$, and $\mathrm{O}$ from the stellar model agree with the nebular ones assuming $t^{2} \neq 0.00$.
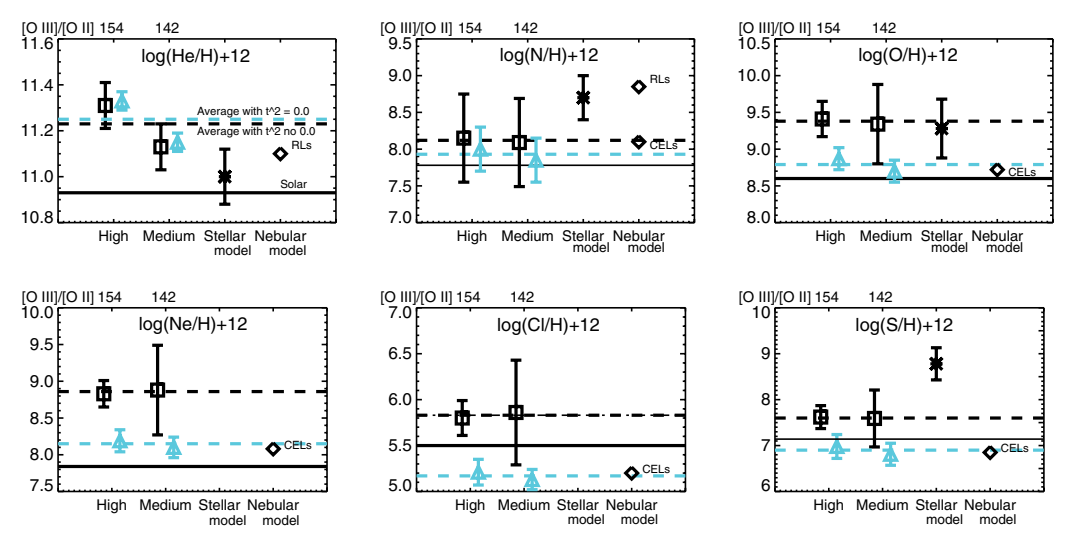

Figure 2. Comparision of the abundances in the nebular regions and models. Triangles are the abundances with $t^{2}=0.00$ and squares are the abundances with $t^{2} \neq 0.00$ in the nebular regions.

\section{References}

Vassiliadis, E. \& Wood, P. R. 1994, ApJS, 92, 125

Hiller, D. J. \& Miller, D. 1998, ApJ, 496, 407

Ferland, G. J., Korista, K. T., Verner, D. A., et al. 1998, PASP, 110, 761 